\title{
Subthalamic Nucleus
}

National Cancer Institute

\section{Source}

National Cancer Institute. Subthalamic Nucleus. NCI Thesaurus. Code C12454.

The paired, biconvex cluster of neurons located under the thalamus that plays a major role in the motor cortico-basal gang lia-thalamo-cortical circuit. The subthalamic nucleus is primary involved in the control of voluntary movement. 